\author{
By Sören Holst \\ Department of Physics, Stockholm university
}

\title{
Physics and theology?
}

d $\mathrm{n}$ his article "Physics and theology" (EPN issue 45/1), John Polkinghorne points out that although physics has been enormously successful in answering questions about physical phenomena, there are many related questions where answers are still lacking. How come we can understand the universe? Could the universe have been in any other way?

I agree with Polkinghorne about the importance of such questions. I also agree that they probably can't be addressed by physics alone. But his suggestion for what field that should complete physics in our search for answers is - theology! This is remarkable, since the issues that he raises are all philosophical, and so most naturally should be dealt with by philosophy (often with essential input from physics). One would therefore expect him to provide some reasons for his point of view. But on this Polkinghorne leaves us without answer.

Or maybe he provides us with a hint. At the end of the section "Cosmic fine-tuning" he refers to religious experience as a kind of legitimate evidence. Could religious experience be what makes him prefer theological explanations over philosophical? Unfortunately, religious experience has never proved to be a source of knowledge. On the contrary, there are innumerable examples of when such experience has led people astray, sometimes in bizarre ways.

One of the issues where Polkinghorne insists that theology could assist physics is the question of cosmic fine-tuning. How can it be that the parameters of our universe seem just right to support the existence of life? Polkinghorne notes that, in the context of quantum gravity, the hypothesis of a multiverse has been suggested. The idea here is that if our universe is just one out of perhaps infinitely many, then surely a few universes will be of the right kind.

Polkinghorne contrasts this hypothesis with another, suggested by theology: a divine Creator. He concludes: "there is no logically coercive principle to settle the choice between these two possibilities".
I see at least three strong reasons to prefer one over the other:

1. One of the hypotheses has explanatory power, the other has not. If the multiverse hypothesis is true, that would really make the question of fine-tuning vanish. This is completely analogous to what has already happened to another similar question: that of the distance between the sun and the earth. Once we realize that there are numerous planetary systems in the universe, we no longer need to ask why the sun-earth distance is so "finely tuned" for life. On the other hand, the hypothesis of a Creator does not clear up any questions. We expect an explanation to refer to a theory which simplifies the phenomena of the world, not to lead to further confusion by introducing ill-defined concepts (such as "divine Creator").

2. One of the hypotheses is part of models suggested to solve also other specific problems in physics, the other is not. Here it is important to realize that the multiverse hypothesis that Polkinghorne refers to is not just some general idea of other possible universes, but rather an implication of some specific inflationary scenarios in the context of quantum gravity. These models are supposed to solve some acute problems in theoretical physics. (Admittedly, these models must be regarded as extremely speculative, and I may add that I myself am not a proponent of any of them.) On the other hand, the hypothesis of a Creator is not part of any model supposed to solve other problems in physics.

3. One of the hypotheses is falsifiable, the other is not. In fact, there is plenty of hypothetical evidence that would disprove any of the suggested models for a multiverse. For example, most kinds of evidence that would falsify general relativity, quantum theory or inflation would also disprove such models. On the other hand, it is hard to imagine any empirical piece of evidence that would make the proponents of the Creator hypothesis abandon it (given that they have not already done so).

One cannot help wondering: Why did the editors of EPN choose to publish an article with such poor scientific analysis? - 